\title{
Scientific research and the human condition
}

\author{
Reviewers are only human, even though they wield enormous power for a moment.
}

Sir — Today I am reviewing an article for a scientific journal. I will generate criticism and express my opinions, and the editor will probably accept them without hesitation, as I am an expert reviewer, if only a god for an instant. But I am only a man, full of inconsistencies and capable of misunderstanding, misrepresenting and making fatal errors.

These facts are often overlooked in the review process. The monstrous machinery we have constructed around scientific research, of which peer review is just one example, does not always augment the quality of research nor make things easier for the average busy researcher who has to fight for funding and prestige in the scientific community (see "Publish and be damned...", Nature 419, 772-776; 2002). After all, fundamental science was done in ancient times, long before the advent of peer review and journal impact factors.

I wonder to what extent my feelings are common to other members of the scientific community when I receive manuscripts or grants for review, and hear a distressed inner voice asking who am I to tell these investigators that what they have done, or want to do, is of no interest to most of the community, and that their work should not be published, or their grant not funded? Whoever is really into research has a modest ego, as we understand our transient existence in a mostly indifferent Universe.

The act of judging — specifically when personal views have to be exposed, such as the 'relevance' I attribute to a particular study for publication in a specific journal — becomes increasingly difficult as I reflect on these issues. The consequences of our extremely involved scientific machinery, and whatever standards we set in our individual or communal research endeavours, are limited by our human condition. We are uncovering fraudulent data, fictional experiments proposed in grant applications that are never performed, rivalry, mistrust and other mischievous behaviour. One sad consequence is the loss of some creative individuals who decide to quit research.

How can we all, as individuals, contribute to a community effort in which everyone - students, technicians and professors alike - can enjoy the pleasures of unravelling the mysteries of nature? Jose Luis Perez Velazquez

Hospital for Sick Children, Division of Neurology, 555 University Avenue, Toronto, Ontario M5G 1X8, Canada

\section{Malaria - there could be a third way}

Sir - The excitement of the simultaneous publication of the malaria parasite Plasmodium falciparum ${ }^{1}$ and mosquito vector Anopheles gambiae ${ }^{2}$ genomes is contagious. Although it is to be hoped that new drugs for malaria are now around the corner, there are some chilling phrases in Nature's bumper issue on Plasmodium genomics ${ }^{1}$. We read that "more people are infected with malaria in Africa today than at any time in history", and that it will require "perhaps two decades to convert genomic information into effective vaccines". A more optimistic view is that all we need to achieve a vaccine is better delivery of known antigens, for example by heterologous priming and boosting.

A stated goal for an effective malaria vaccine is to "induce protective immune responses equivalent to, or better than, those provided by naturally acquired immunity or immunisation with attenuated sporozoites". Why, then, is there no attenuated sporozoite vaccine? Part of the answer is that sporozoites cannot easily be grown in vitro. Proteomic analysis, with small quantities of sporozoites from infected mosquitoes, has neatly resolved the problem of analysing sporozoite gene expression. Astonishingly, this has shown that sporozoites express many genes of the var and riffamilies, which are largely responsible for antigenic variation. This is encouraging for vaccine development, and poses several obvious questions. Are the antigen genes expressed in sporozoites representative of var gene diversity or are they at specialized genomic sites? Do the sporozoite antigens change with sequential cycles of a single strain? How divergent are the sporozoite repertoires of different strains?

The var and rif antigenic diversity of sporozoites could stimulate new investment in seeking cultivation technology for the entire Plasmodium life cycle. The $P$. falciparum and $A$. gambiae genomes are littered with clues as to what the parasite needs to thrive. There have been some notable preliminary successes with in vitro systems. A relatively simple culture system ${ }^{3}$ has been described for growing oocysts and sporozoites of $P$. falciparum, but does not seem to have been exploited. More recently, a similar system has been achieved for P. bergheit .

It would be foolish to suggest that all that is needed to obtain a malaria vaccine is to grow sporozoites in bulk. The protection attained with X-irradiated sporozoites, or X-irradiated infected mosquitoes, was a benchmark, but resistance faded and challenge broke through in some volunteers ${ }^{5}$. Var gene diversity is generated by recombination, and the repertoire of the blood-form stages appears to be immense ${ }^{6}$.

Targeted regional vaccines are unlikely to work because antigenic types do not cluster geographically. There is no guarantee that sporozoite pools can be generated with adequate antigenic repertoires, although immune pressure in vitro might be used to improve the yield of var gene recombinants. Nevertheless, a determined sporozoite cultivation consortium initiative would cost little and should pay rich dividends. New means of sporozoite attenuation and delivery could be explored.

Michael A. Miles

Department of Infectious and Tropical Diseases, London School of Hygiene and Tropical Medicine,

Keppel Street, London WC1E 7HT, UK

1. Gardner, M. J. et al. Nature 419, 498-511 (2002).

2. Holt, R. A. et al. Science 298, 129-149 (2002).

3. Warburg, A. \& Schneider, I. Exp. Parasitol. 76, 121-126 (1993).

4. Al-Olayan, E. M. et al. Science 295, 677-679 (2002).

5. Richie, T. L. \& Saul, A. Nature 415, 694-701 (2002).

6. Fowler, E. V. et al. Mol. Biochem. Parasitol. 120, 117-126 (2002).

\section{Academics are teachers and colleagues too}

Sir - The proposal in Correspondence by E. Fernández-Juricic et al. (Nature 420, 16; 2002) to apply impartial peer review to academic hiring ignores several important factors. One is the central role of academic institutions - to educate.

At most academic institutions, faculty members teach as well as conduct research. Merely assessing research qualifications is no indication of the ability to communicate complex ideas or to empathize with students. Personality also looms large, particularly in small departments - the highly qualified eccentric who cannot interact with people may be a difficult and ineffective colleague.

In their zeal to provide dispassionate assessment, Fernández-Juricic et al. seem to have forgotten that science is a human activity. My hope is that their proposal was made tongue-in-cheek.

\section{Karen F. Greif}

Department of Biology, Bryn Mawr College, Bryn Mawr, Pennsylvania 19010, USA 